\title{
Investigating the Effects of Signal Light Position on Human Workload and Reaction Time in Human-Robot Collaboration Tasks
}

\author{
Teegan Johnson ${ }^{1}$, Gilbert Tang ${ }^{1}$, Sarah R. Fletcher ${ }^{1}$ and Phil Webb ${ }^{1}$ \\ ${ }^{1}$ Centre for Advanced Systems, School of Aerospace, Transport, and Manufacturing, \\ Cranfield University, United Kingdom \\ \{t.1.johnson, c.tang, s.fletcher, p.f.webb\}@cranfield.ac.uk
}

\begin{abstract}
Critical to a seamless working relationship in human- robot collaborative environments is effective and frequent communication. This study looked to assess whether placing a light source on a robot was more effective for informing the human operator of the status of the robot than conventional human-machine interfaces for industrial system signaling such as light towers. Participants completed an assembly task while monitoring a robot and changes to the light sources: either from one of two light towers or LED strip lights attached to the robot. Workload was assessed by measuring reaction times to light changes and by counting number of completed assemblies. Although both the ANOVA and Friedman tests returned none significant results, total misses per condition showed that the participants did not miss any of the robot lights, whereas signals were missed for the light towers.
\end{abstract}

Keywords: Human-Robot Communication · Collaboration · Human Factors Eye Tracking

\section{$1 \quad$ Introduction}

It is not currently feasible to fully automate all processes completed by human operators within manufacturing, as a result of limitations in both automation and robot systems. The limitations and strengths of both humans and robots have been detailed in function allocation models, particularly Fitts List [1] which still persists in its usefulness today [2]. As a result of these differences in capabilities, humans and robots can complement each other in areas where full automation is not possible [3]. This may include robots supporting human operators in their work, such as holding heavy objects that cannot be safely lifted by an operator, or completing fetching and carrying parts or tools. Unhelkar and colleagues [3] provide an example of this in their study which looked to introduce a mobile robotic assistant that delivers tools and materials to workers in an automotive manufacturing plant. By complementing the weaknesses of a human operator with the strengths of a robot device and vice versa, production efficiency can be improved.

Critical to seamless collaboration in this working relationship is effective and frequent communication between the robot system and the human co-worker. Endsley's 
[4] model of situation awareness highlights this; it states that the first stage of processing before making a decision is the operator's perception of elements in the current system. If this information is wrong or miss understood, the wrong decisions are made. Poor control and display design has been implicated in many accidents [5]. Furthermore, within both the fields of aviation and transport a loss of situational awareness due to attentional and perceptual errors were identified as significant causal factors $[6 ; 7 ; 8]$. Therefore, learning from this research, communication in collaborative robotic tasks needs to be both effective and efficient. Particularly where the robot and human operator truly work together on a task and therefore working in close proximity to each other.

Because manufacturing plants are noisy environments, visual systems are employed to provide information to operators regarding the status of machinery. Some of the current indication methods used within manufacturing for automated and robot systems are light towers (as seen in figure 1), industrial light towers use the traffic light system to inform operators or mangers of the state of the system. They are stationary light sources that are generally placed on top of fully automated systems so that a single manager can easily assess the state of multiple systems [9].

The original design application for light towers does not lend itself to effective communication between a human operator and a robot during collaborative tasks. The standards in place that dictate the best placement of light towers [10], combined with the need to place the tower light outside of the robot's working area to prevent it from hindering the robot's movements. These factors lead to light tower placement at a distance from the robot, and may place the light tower in the periphery of the operator's vison. This places the light tower in the peripheral vision of operators, which may result in missed signals as a result of the limitations of peripheral vision with regards to static objects [11]. This is a concern in highly collaborative environments because it may mean that the operator's attention is split three ways (between their task, the robot completing its task and the light tower). Additionally during a collaborative task the light tower may be ignored because the robot is the main area of interest. This may lead to lower task efficiency, and missed signals which could lead to errors, mistakes and potentially accidents.

A solution to this is to reduce the number of areas vying for the operator's attention, by placing the lighting on the robot. As a result the warning system would be larger in size than a light tower, attached to the object of interest within the collaborative work, and will be moving. All of which help to increase the detectability of the light, movement is known to enhance the visibility of objects, particularly in the peripheral vision where stationary objects may not be seen [11]. Therefore the expected effects for the human co-worker are lower workload, faster reaction time and fewer missed signals. However an investigation was required to examine these assumptions and to identify whether the attachment of strip lighting to the robot would have a positive impact for the human co-worker, compared to a traditional industrial light tower.

Consequently the aim of this research is to assess the effectiveness of attaching an indication system to a collaborative system, compared to traditional light tower. 


\section{Method}

\subsection{Participants}

10 participants, 6 female and 7 male, were recruited using random sampling (age range: 22-58 years, mean: 32.9 , SD: 13.71$)$.

\subsection{Design}

A within-subjects $3 \times 2$ experimental design was used to investigate the effect of indication light sources (independent variables) on reaction time and workload (dependent variables). The three conditions are two light towers (Harmony XVC6, Schneider Electrical) (one placed at a traditional distance and the second placed opposite the robot) and 5050 RGB LED strip lights placed on a UR5 robot (Universal Robots A/S). The second light tower was placed opposite the robot to identify whether distance between the light tower and the participant affected the dependent variables. All participants completed an assembly task and reacted to the light sources by pressing a button used to measure reaction time.

\subsection{Apparatus and Laboratory Set Up}

The experiment took place in a 3960mm x 3900mm laboratory area surrounded by 4 sides of wall, with no direct sunlight to ensure that the lighting level was kept at a constant 400 lux throughout the entire experiment. Figure 2 shows the laboratory set up, the robot used was a single armed UR5 robot that completed a pick-up and place task using PVC pipes on a worktop directly in front of it. As can be seen in figure 1 LED lights were wrapped around the UR 5, covering the area between the elbow and wrist and the wrist and base.

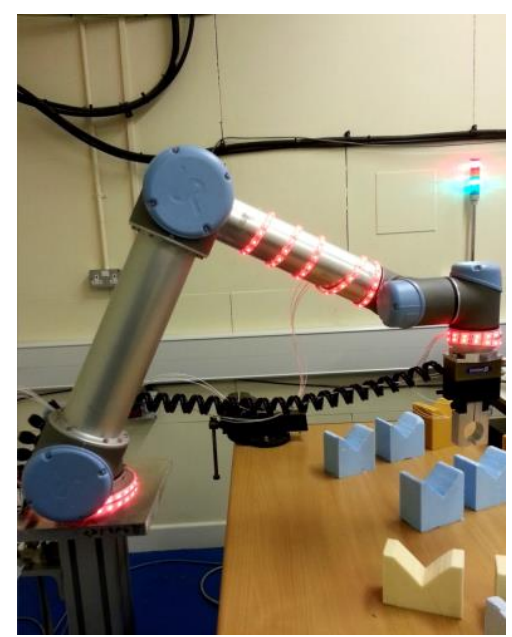

Fig. 1. UR 5 with LED wrap around lights 
Two industrial light towers were used; one was placed in a location replicating current manufacturing placement, and using the British Standards Institute's standards for the set up requirements of indication systems for industrial machinery [12 and 10]. It was postitioned at a height of $1210 \mathrm{~mm}$, and ensured that it was within 10 degrees of the horizontal line of site, and not obstructed by the robots movements. The second light tower was placed directly opposite the robot, at an equal distance from the central line of the worktop used by the robot. For simplicity, only the green and red lights were used on the indicating devices. Both green and red lights on the tower lights emitted 1300 lux while the LED light strip emitted 600 lux in green and 230 lux in red due to the limitation of the lighting system.

As can be seen in figure 2, a workbench was set up in front of the robot worktop at the same height, for the participant to complete the assembly task on. On the worktop, three containers were placed on the table each holding, nuts, washers and bolts, these could be moved around by the participants for ease of use. A button to measure reaction time was attached to the table on the left hand side. This button was connected to a National Instruments logging system to record participants' reaction time and any misses of the light sources.

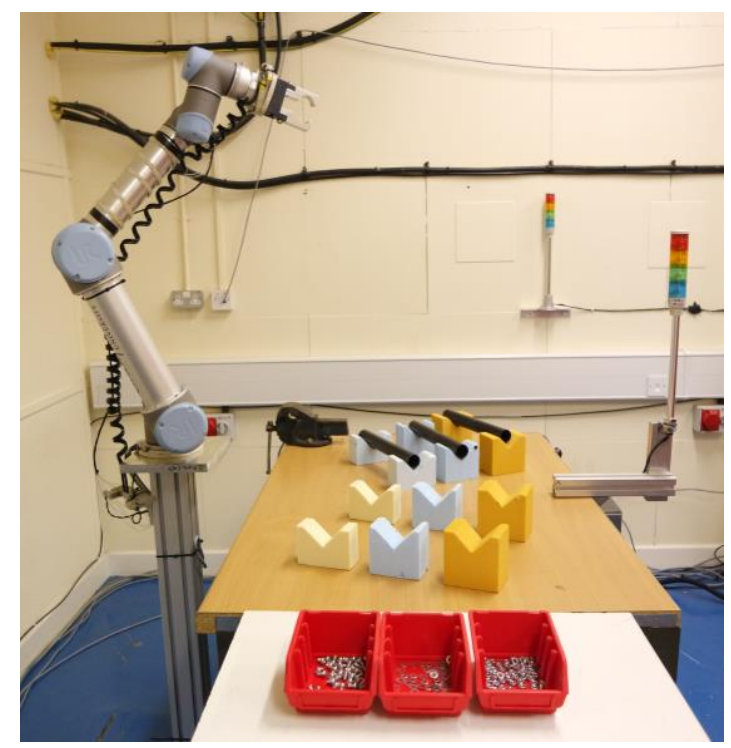

Fig. 2. Experimental cell layout

\subsection{Procedure}

After being briefed about the experiment participants were lead into the laboratory area and fitted with a pair of head-mounted eye-trackers (ETG-1.7, SensoMotoric Instruments). To minimize learning effects the participants were asked to complete a pre-task assembly; this involved the participants completing 10 assemblies as quickly as possible while being timed. The assembly task for the experiment involved threading a washer onto a bolt and then attaching a nut to the bolt. Upon completion of the 
assembly participants were familiarized with the robot movement and the light sources.

The task was then explained to the participants; they were informed that their task was to complete as many assemblies as possible while monitoring the light sources. If they saw a green or a red light they were to press the button on their left hand side, which would turn the light off. If the robot did anything unusual, the participants were asked to make a mental note of it. It was emphasized that their primary aim was to complete as many assemblies as possible, while staying aware of what was happening around them. The participants completed this activity three times, with a different light source used in each task.

Cyclical counterbalancing was used for the presentation of the light sources to prevent practice effects [13]. After each task they were asked three questions regarding the task they had completed, their assessment of the light source, and the robots movement. This was to enable the researcher to remove the completed assemblies for counting, and to gain an understanding of how the participant found the task and the light source. Upon completion of the final task, participants were thanked for their time and debriefed about the aim of the research.

\subsection{Analysis}

The eye trackers were used to capture participants visual dwell times while taking part in the experiment, this data was analyzed using Begaze software (SensoMotoric Instruments) utilizing Area of Interest (AOI) semantic gaze mapping. The AOIs that were mapped included the manual task work top, robot work top, and the UR5. Workload was assessed by capturing reaction times or misses of the light signals, and by counting the number of assemblies completed per condition.

\section{Results}

The study looked to investigate the effectiveness of placing an indication light on a robot compared to a traditionally placed light tower, and a light tower placed in line with the robot.

Normality tests were applied to dwell time for the manual workbench, the robot work bench and the robot, across each of the light conditions. None of the data sets were normally distributed; therefore the Friedman Test was applied to the data. No significant differences were found between in length of dwell time as a result of indication light conditions (manual work bench: $\chi 2(2)=5.600, p>0.05$; robot workbench: $\chi 2(2)=3.800, p>0.05$; robot: $\chi 2(2)=4.200, p>0.05)$. The total dwell time results are presented in table 1. Results show that the total dwell time on the robot and the manual worktop is longest in the robot light scenario (236924.5ms). However, dwell time on the robot workbench is highest $(453196.3 \mathrm{~ms})$ when the nearer tower light was used. 
Table 1. Dwell Time Total (ms); TL=Tower Light

\begin{tabular}{llll}
\hline & TL $($ Wall $)$ & TL (Bench) & Robot Light \\
\hline Robot & 171176.8 & 166486.9 & 236924.5 \\
Robot workbench & 318387 & 453196.3 & 276114.5 \\
Manual worktop & 149189.5 & 119214.2 & 185870.1 \\
\hline
\end{tabular}

A repeated measures ANOVA with a Greenhouse-Geisser correction determined there was no significant main effect of light source on reaction time $(\mathrm{F}(1.178,10.606)$ $=2.830, \mathrm{p}>0.05)$. The reaction time and number of misses for each participant are presented in table 2 .

Table 2 presents the reaction time and total number of misses for each participant. As can be seen none of the participants missed any of the robot light signals, but the total missed signals for the tower lights are 13 and 8 in the wall mounted and bench positions respectively. The robot light had the shortest total reaction time (13.45s) whilst the wall mounted tower light yielded the longest reaction time (14.74s).

Table 2. Reaction Time (RT) and number of misses

\begin{tabular}{lllllll}
\hline \multirow{2}{*}{ Participant } & \multicolumn{2}{c}{ TL (Wall) } & \multicolumn{2}{c}{ TL (Bench) } & \multicolumn{2}{c}{ Robot Light } \\
& RT (s) & Misses & RT (s) & Misses & RT (s) & Misses \\
\hline 1 & 1.72 & 1 & 1.46 & 1 & 1.46 & 0 \\
2 & 1.35 & 1 & 1.56 & 1 & 1.42 & 0 \\
3 & 1.38 & 2 & 1.50 & 0 & 1.48 & 0 \\
4 & 1.51 & 0 & 1.54 & 2 & 1.53 & 0 \\
5 & 1.15 & 0 & 1.27 & 0 & 1.06 & 0 \\
6 & 1.21 & 0 & 1.20 & 0 & 1.21 & 0 \\
7 & 1.47 & 1 & 1.38 & 1 & 1.28 & 0 \\
8 & 1.45 & 1 & 1.15 & 0 & 1.13 & 0 \\
9 & 1.57 & 5 & 1.51 & 3 & 1.40 & 0 \\
10 & 1.92 & 2 & 1.42 & 0 & 1.48 & 0 \\
Total & 14.74 & 13.00 & 14.01 & 8.00 & 13.45 & 0.00 \\
Mean & 1.47 & 1.30 & 1.40 & 0.80 & 1.34 & 0.00 \\
SD (2 d.p) & 0.23 & 1.49 & 0.15 & 1.03 & 0.17 & 0.00 \\
\hline
\end{tabular}

Between each condition the participants were asked how they found monitoring the light source and at the end of the experiment were asked which light source they preferred out of all of the options and why. Although no significant differences were found in dwell time and reaction time between the light sources there was a preference for the robot light over the two light sources for the participants. Eight of the ten participants preferred the robot light source (Participants, 2, 3, 4, 5, 7, 8, 9, and 10), the reasons given were that the movement (Participant 2), size of the light source on 
the robot (Participants 2 and 9), proximity (Participant 3), and the fewer areas requiring attention (Participant 4) meant that participants felt they were able to see the light source with greater ease than the light towers. Additionally participants 5 and 8 stated that the light at the base of the robot made it easier for them to see light signals while they were paying attention to the assembly task, because it was within their line of sight.

Participant 1 found the robot light too bright and therefore distracting; and participant 6 had no preference other than they found the wall light task more difficult because it was further away, participant 2 and 9 had the same responses. Three of the participants (Participants 3, 4, and 7) stated that they had used their peripheral vision to register light changes, which would not have been picked up as dwell time lengths on the light sources.

\section{Discussion}

Participants were asked to focus on a manual task and react to any light signal, while paying attention to the activity being completed by a robot. This activity was chosen because it reflects current industrial practices providing the light task with a level of validity. Although the statistical analyses were not significant the eye tracking results seen in Table 1 revealed that the participants paid more attention to the robot and the manual task when the light signal to be monitored was positioned on the robot. On the other hand, robot workbench receives more attention when the light signals were coming from a tower light positioned next to it. In comparison, the participants' attentions were extended to the other two AOIs when the wall mounted tower light was used. This could be an indication that when the robot light signals divert less users' attention away from important AOIs.

The reaction time to light signal was generally shorter when the robot light was activated when compare to the tower lights. The robot light has also received a hundred percent hit rate whilst over half of the participants have missed at least one of the tower light signals. This shows that participants could observe the robot light more easily while carrying out a manual task. Furthermore, it was noticed from the eye tracking recording that most participants relied on their peripheral view to observe light signals from either of the tower lights. This is corroborated with the interview data, 3 of the participants stated they had made a conscious effort to use their peripheral vision to track the lights. This aligns with previous research that stationary objects in peripheral are more likely to be missed [11].

Further research is required with a larger sample size, and a complex task. The current task was simple and enabled participants to complete the assemblies without paying visual attention. Therefore the cognitive load from the activity was low, and meant that participants were able to use their remaining available cognitive resources to monitor the lights and the robot activities. A task requiring more attention may see a change to the results, with an increase in the number of light signals missed. Although the overall aim of this activity was to assess the effectiveness of light sources in human-robot collaborative environments, the task is one of proximity not collaboration one. The reason for this this was used to establish a bench mark for attention before progressing towards a collaborative activity. 
Acknowledgments. The authors would like to thank John Thrower for his support during the research. This work was supported by the UK Engineering and Physical Sciences Research Council as part of the Centre for Innovative manufacturing in Intelligent Automation under the grant reference number EP/1033467/1.

\section{References}

1. Fitts P. M., (ed): Human engineering for an effective air navigation and traffic control system. National Research Council (1951)

2. de Winter, J.C.F., Dodou, D.: Why the Fitts list has persisted throughout the history of function allocation. Cogn. Tech. Work. 16, 1--11 (2014)

3. Unhelkar, V. V., Siu, H. C., Shah. J. A.: Comparative performance of human and mobile robotic assistants in collaborative fetch-and-deliver tasks. In Proceedings of the 2014 ACM/IEEE international conference on Human-robot interaction (HRI '14), pp. 82--89. ACM: New York (2014)

4. Endsley, M. R.: Measurement of situation awareness in dynamic systems. Human Factors. 37, 65--84 (1995)

5. Sanders, M. S., McCormick, E. J.: Human Factors in engineering and design. McGrawHill, NewYork (2007)

6. Matthews, G., Davis, D. R., Westerman, S. J., Stammers, R. B.: Human Performance: Cognition, stress and individual differences. Psychology Press, Hove (2000)

7. Shinar, D.: Psychology on the road: the human factor in traffic safety. Wiley, Chichester (1978)

8. Endsley, M. R. Automation and situation awareness, in R. Parasuraman and M.Mouloua (eds), Automation and human performance: Theory and applications (Mahwah, NJ: Lawrence Erlbaum Associates), 163-181. (1996)

9. Greif M.: The visual factory: building participation through shared information. Productivity Press (1991)

10. British Standards Institution, BS EN 61310-1 Safety of machinery. Indication, marking and actuation. Requirements for visual, acoustic and tactile signals (2008)

11. Breedlove, S. M., Rosenzweig, M. R., Watson, N. V.: Biological Psychology: an Introduction to Behavioral, Cognitive, and Clinical Neuroscience. Sinauer Associates, Inc, Sunderland (2007)

12. British Standards Institution, BS EN 60073 Basic and safety principles for man-machine interface, marking and identification. Coding principles for indicators and actuators (2002)

13. Keppel, G., Saufley, W. H., Tokunaga, H.: Introduction to Design and Analysis A students Handbook. Freeman, New York (1992) 


\section{Investigating the effects of signal light position on human workload and reaction time in human-robot collaboration tasks}

Johnson, Teegan L.

Springer

Investigating the Effects of Signal Light Position on Human Workload and Reaction Time in Human-Robot Collaboration Tasks, Advances in Intelligent Systems and Computing, Advances in Ergonomics of Manufacturing: Managing the Enterprise of the Future: Proceedings of the pÿAHFE, Christopher Schlick, Stefan TrzcieliDski.

http://dx.doi.org/10.1007/978-3-319-41697-7_19

Downloaded from Cranfield Library Services E-Repository 